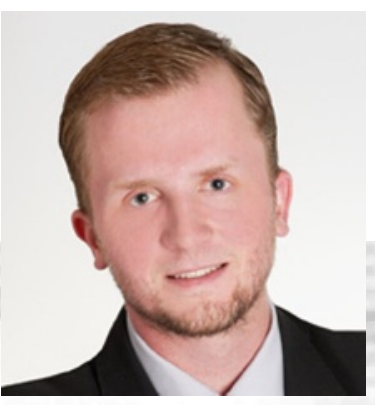

Sebastian Feld Koordinator "Innovationszentrum Mobiles Internet", LMU München

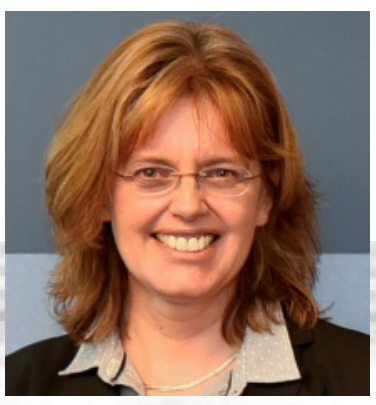

Prof. Dr. Claudia Linnhoff-Popien Lehrstuhl "Mobile und Verteilte Systeme", LMU München

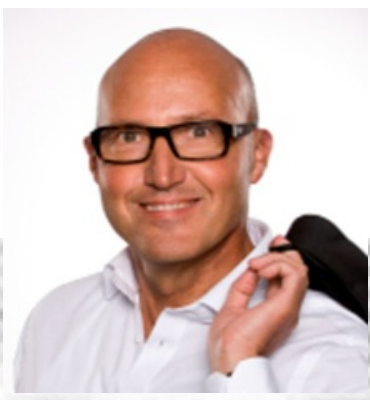

Rainer Göttmann Geschäftsführer metafinanz Informationssysteme $\mathrm{GmbH}$

\title{
Wo bleibt die Sehnsucht nach dem Neuen?
}

\section{Alles wird $\mathbf{4 . 0}$}

Die Industrie, die Arbeit, die Dienstleistungen.

Mit anderen Worten: Es geht voran.

Wir haben gelernt, und wir verbessern uns kontinuierlich. Aber selten mit solch starken Veränderungen, dass der Wandel sogar eine Versionsnummer erhält.

Und wie reagieren wir?

Wie immer!

Wir sind skeptisch, suchen nach dem Haken an der Sache, fühlen uns überrollt, möchten doch lieber erst einmal abwarten ... Gleichzeitig sind wir verzweifelt, denn wir spüren, dass unser Wunsch nach Stabilität - man kann es auch Stillstand nennen - in die falsche Richtung zielt. Kaum jemand würde heute noch behaupten, dass 4.0 aufzuhalten ist. Wer nicht mitfährt, kann sich nur noch hinter den Zug werfen. Es ist ja nicht so, dass wir das nicht wüssten ...

4.0 steht für die Zukunft, für den Abschied von der Vergangenheit - wir schließen das Kapitel Automatisierung und öffnen ein neues mit der Überschrift Digitalisierung. Nach der Dampfmaschine, dem Fließband und der Computertechnik ist nun die nächste industrielle Revolution eingeläutet. Es knistert an allen Ecken und Enden, unzählige Startups probieren sich gerade in verschiedenen Industrien aus. Das Spektrum der digitalen Ökonomie erstreckt sich über sämtliche Bereiche des Wirtschaftens und Lebens. Ein Blick in die Startup-Szene zeigt Beispiele aus allen Bereichen: Sei es Klima und Energie, Gesundheit und Ernährung, Mobilität, Sicherheit oder Kommunikation. Innovative Gründungen finden sich darüber hinaus in Clustern wie Bio- und Medizin-Technik, Stadtplanung, sowie in den „,klassischen" Branchen à la Handel, Logistik oder Tourismus.

Diese thematische Breite ist der Indikator dafür, dass wir es keineswegs mit einem Hype oder Strohfeuer zu tun haben. Wir stehen vor einem Strukturwandel, der sämtliche Stufen der Wertschöpfung von der Produktion bis zur Dienstleistung erfasst und vernetzt, ganze Märkte verändert und nicht zuletzt auch neue Umsätze verspricht.

\section{Kern dieser Revolution ist die Vernetzung}

Technologisch ist die Basis gelegt: Leistungsstarke Verbindungen, Cloud-Systeme und die schnelle Verarbeitung großer Datenmengen sorgen dafür, dass Unternehmen neu denken und agieren können. Auch, weil sie in immer engerem Austausch zueinander stehen. Die Szenarien sind schon da: Maschinen sprechen mit Maschinen, Sensoren tauschen sich untereinander aus, Kühlschränke schicken Nachrichten an Supermärkte, Autos verknüpfen sich mit Versicherungen - die Beispiele, um die Zukunft in einer gelingenden Vernetzung zu präsentieren, sind unzählig und nehmen Tag für Tag zu.

Doch die Herausforderung des Neuen liegt nicht allein in der Technik, auch wenn der Begriff Digitalisierung dies gerne suggeriert. Sie liegt in unserer Fähigkeit, uns zu vernetzen. Erfolg oder Scheitern wird davon abhängen, inwieweit Menschen und Unternehmen lernen, sich nicht mehr als Zentrum des Geschehens zu betrachten, sondern als Teil einer eng verwobenen Struktur, in der viel mehr mit- als gegeneinander gehandelt wird.

Schlagworte wie Co-Creation, Open Innovation, Transparenz oder Agilität stehen daher letztlich nicht für die Nutzung von Technik, sondern fordern Veränderungen in den Organisationen, in den Prozessen, vor allem aber 


\section{$\{$ EDITORIAL}

in den Köpfen. Denn weder 10T, M2M, Enterprise Chat noch Industrie 4.0 machen ein Unternehmen erfolgreich, sondern das, was Menschen daraus machen.

Entsprechend wird auch die wichtigste Voraussetzung sein, den Wandel in den Köpfen herbei zu führen. Stellen wir also den Menschen in den Mittelpunkt: als Manager, Mitarbeiter, Kunden und als Geschäftspartner.

\section{Management 4.0}

Gefordert sind in besonderem Maße die Entscheider und Unternehmenslenker. Sie müssen auf der einen Seite Orientierung geben, denn noch herrscht viel Unsicherheit, Unplanbarkeit und Unwissenheit. Gleichzeitig sind sie selbst gefragt, sich zu verändern, denn die klassischen hierarchischen Arbeits- und Denkweisen, wie sie in Deutschland vorherrschen, haben ausgedient. Vernetzungsintelligenz und empathisches Handeln werden zu den entscheidenden Fähigkeiten für erfolgreiche Führung. Ebenso wie der sichere Umgang mit den neuen Medien zu den Basiskompetenzen gehört, die den Aufbau und die sorgfältige Pflege der unabdingbaren Netzwerke überhaupt erst ermöglichen. Persönliche Kontakte werden zur Pflicht.

\section{Der Mitarbeiter 4.0}

Von uns allen werden neue Fähigkeiten verlangt: Vernetztes Denken, Überblickswissen, Kommunikationsfähigkeit und nicht zuletzt IT-Kenntnisse werden immer wichtiger. Beispiel Smart Fabric: Im neuen Industriezeitalter nimmt der Bedarf an Hoch- und Höchstqualifizierten zu. Mitarbeiter werden künftig gebraucht, um Probleme zu lösen und Entscheidungen zu treffen und die vernetzte Fabrik am Laufen zu halten. Sie müssen Apps bedienen können oder sich mit Datenbrillen durch die Fabrik bewegen.

\section{Der Kunde 4.0}

Die neuen, digitalen Möglichkeiten verändern die Anforderungen - auch Kundenwünsche genannt. Produkte müssen einerseits einfacher zu handhaben sein, andererseits aber immer Komplexeres leisten, während sie so individuell wie möglich auf die Bedarfe ausgerichtet sind. Solchen Wünschen kann kein Fachmann mehr allein entsprechen. Dafür braucht es eine neue, engere Form der Zusammenarbeit innerhalb der Firmen und zwischen den Unternehmen. Experten verschiedener Disziplinen und Branchen müssen sich austauschen, ihre Kenntnisse zusammenbringen.

\section{Geschäftspartner 4.0}

Die richtige Balance zu halten, dürfte zu einer der großen Herausforderungen im geschäftlichen Miteinander werden. Nähe ist wichtig und eine zwingende Folge von mehr Vernetzung, größerer Transparenz, engerer Zusammenarbeit. Aber auch Distanz muss gewahrt werden. Ein Geschäftspartner ist kein Kollege. Ein Lieferant ein anderes Unternehmen. So einfach es heute ist, rund um den Globus neue Geschäftsbeziehungen aufzubauen oder bestehende zu vertiefen, so flüchtig können diese Verhältnisse sein. Gerade durch die zu erwartende Stärke von Marktplätzen und unternehmensübergreifenden Plattformen werden sich in Zukunft noch mehr Player auf dem "Spielfeld" begegnen. Für eine Phase eines Projektes kann eine enge Zusammenarbeit stattfinden, für den nächsten Schritt kann diese schon wieder mit anderen Partnern stattfinden. Doch auch hier wird die menschliche Komponente eine wichtige Rolle spielen. Wer eine Partnerschaft eingeht, wird sich letztlich nicht allein auf technische Möglichkeiten, Zahlen und Daten verlassen, sondern will seinem Gegenüber in die Augen schauen und ein Gefühl für ihn entwickeln. Das Miteinander im Geschäftsleben wird auch angesichts einer vierten Industrierevolution nicht von einem Jahrhunderte alten Prinzip abweichen: dem Bauchgefühl als Basis von Entscheidungen.

\section{Wir brauchen also einen Fortschritt 4.0}

Die aktuelle Entwicklung fordert ein anderes und nicht zuletzt auch schnelles Handeln, denn Geschwindigkeit ist das Gebot der Stunde. Statt abzuwarten (und abgehängt zu werden), muss die Bereitschaft entstehen, andere Wege zu gehen, Neues auszuprobieren und Risiken zu wagen. Gesagt haben wir es oft genug, aber in einem Umbruch wie wir ihn jetzt erleben, müssen wir lernen. Lernen, eine andere Haltung anzunehmen, lernen, uns zu öffnen, lernen, Risiken einzugehen. Gerade heute, am Beginn einer neuen Entwicklung, wird es wichtig sein, Scheitern nicht als Makel, sondern als Chance zu begreifen. Es beim nächsten Mal (noch) besser zu machen, ist leichter gesagt als getan. Aber es ist alternativlos. 
Gestalter des Wandels zu werden und nicht in Ehrfurcht vor den anstehenden Veränderungen zu erstarren, ist eigentlich ganz leicht. Lassen Sie uns den Blick vor allem auf die positiven Seiten richten. Auf die Chancen und Opportunitäten, die Verbesserungen und Erleichterungen, die 4.0 bringen wird. Optimismus und Träumen ist erlaubt. Vielleicht entstehen so ja auch die Sehnsucht nach dem Neuen und die Lust an der Veränderung.

Viel Freude beim Lesen und den ein oder anderen inspirierenden Gedanken wünschen

\section{Ihre}

\section{Sebastian Feld, Claudia Linnhoff-Popien und Rainer Göttmann}

